\title{
Effect of Anthelmintics on Antioxidant Enzymes in Arabidopsis Thaliana
}

\author{
Radka Podlipná ${ }^{1}$, Eliška Syslová ${ }^{1,2}$, Markéta Hanulíková ${ }^{2}$ Lucie Raisová Stuchlíková $^{2}$, \\ Barbora Szotáková ${ }^{2}$ Lenka Skálová ${ }^{2}$ \\ ${ }^{1}$ Laboratory of Plant Biotechnologies, Institute of Experimental Botany, Czech Academy of Sciences \\ Rozvojová 263, Praha 6, Czech Republic \\ ${ }^{2}$ Department of Biochemical Sciences, Faculty of Pharmacy in Hradec Králové, Charles University in Prague \\ Heyrovského 1203, Hradec Králové, Czech Republic \\ podlipna@ueb.cas.cz; lenka.skalova@faf.cuni.cz
}

\section{Extended Abstract}

Veterinary drugs have potential to enter the environment and cause adverse ecological or human health effects. Although the health-risk assessment of these pharmaceutical compounds regarding their toxicity has been studied for a long time, little is known about the ecotoxicological effects on non-target organisms. Since veterinary drugs, as biologically active compounds, are designed to interact with a target molecule in the animal, in the environment they may affect other organisms having the same target or they can exert toxicity via other mechanisms [1].

In our project we focused on the changes in plant antioxidant system after contact with veterinary anthelmintics. We used in vitro suspension cultures of thale cress (Arabidopsis thaliana) as model system. Cell suspensions were incubated with anthelmintics monepantel (MOP), albendazole (ABZ), fenbendazole (FBZ), flubendazole (FLU) and ivermectin (IVM) for various times (from 8 hours to 3 days). After incubation, the cell suspensions were collected and the activity and expression of the main antioxidant enzymes were measured as the changes in the activities/expression of the antioxidant enzymes represent a widespread response observed in plants under various stresses.

The results showed the significant increase of peroxidase activity (POX) after ABZ, FLU, FEN and IVM treatment of Arabidopsis thaliana suspension cultures while catalase activity increased only in cells treated by ABZ and MOP. The activity of ascorbate peroxidase (APX) and glutathione S-transferases was not affected by any tested anthelmintics with exception of IVM, which significantly increased the activities of APX. In addition, IVM also markedly increased POX activities (by 20-fold) after 8 and 24 hour -treatment. Certain changes in the expression of antioxidant enzymes were observed too.

In conclusion, veterinary anthelmintics can induce stress in plants and might affect their antioxidant systems.

This project was supported by Czech Science Foundation, grant No. 15-05325S.

\section{Reference}

[1] H. Bártíková, L. Skálová, "Veterinary drugs in the environment and their toxicity to plants," Chemosphere, vol. 144, pp. 2290-2301, 2016. 